\title{
Patients' Perceptions of Health Information Given by Nurses and Clinicians at Discharge at Chiradzulu District Hospital in Malawi, Sub-Saharan Africa
}

\author{
Reuben Solomon Mumba, (RN, Bsc. Nurs, MPH) \\ St. Joseph's College of Nursing, Malawi.
}

Doi: 10.19044/esj.2018.v14n18p65 URL:http://dx.doi.org/10.19044/esj.2018.v14n18p65

\begin{abstract}
Background: Hospital discharge information is a critical component of preparation to facilitate patient transition from hospital to home. Numerous studies elsewhere provide evidence that patients and families encounter a variety of problems after discharge from hospital such as difficulties with functional abilities and carrying out personal care. These problems are often attributed to having unmet informational needs at discharge. Objective: The main objective of the study was to assess patients' perceptions of the health information provided by nurses and clinicians at discharge. Methods: The study was conducted at Chiradzulu District Hospital in Malawi. A qualitative method of data collection (in-depth interviews) was used. Convenience sampling method was used to select patients into the study. Data was analyzed using Nvivo 9 software. Findings: The study found that most patients were given information by nurses and clinicians on how to perform self-care at home on discharge from hospital. The study revealed that most patients perceived the discharge information as relevant in assisting them with home care. In addition, the study found that most patients perceived it as inadequate. The study also found that the factors that facilitated patients understanding of information were clear explanation of information and use of understandable language by information providers. Barriers included lack of encouragement from information providers to make patients speak, incomprehensive information provided to the patients and patients fear of asking information providers' questions. Conclusion: Nurses and clinicians should make sure that all patients are given some discharge information to help them to manage their self care at home. Those patients who are at high risk for readmission should be given detailed information.
\end{abstract}

Keywords: Patient's perception, health information, patient's dischage 


\section{Introduction}

Going home following hospitalization has commonly been referred to as a transition for the patient and the family that begins prior to discharge and extends into the post-discharge period (Smith \& Liles, 2007). A transition is a process of passage from one life phase, condition or status to another during which changes in health status, role relations, expectations or abilities create a period of vulnerability (Meleis et al., 2000). Transition theory proposes components of transition such as the nature of the transition, transition conditions and nursing therapeutic practices affecting patterns of response during a transition. The nature of the discharge transition is influenced by the personal (patient characteristics) or environmental conditions (factors associated with hospitalization) that facilitate or hinder progress toward achieving a healthy transition outcome (Meleis \& Trangestein, 1994). Patient knowledge at discharge on how to carryout self-care at home is one of the transition conditions that can facilitate or hinder progress. Feeling confident and connected to supportive networks, including the health care system, are key response patterns and evidence of sufficient transition (Meleis \& Trangestein, 1994). Patient readiness for discharge is identified as an indicator of these response patterns for the patient transition situation.

Studies have shown that patients face problems in carrying out their personal care after they are discharged from hospital (Tierney et al., 1993; Selman, 2011). These problems are often attributed to unmet informational needs (Jacobs, 2000). Malawi is one of the countries in sub-Saharan Africa that have a high re-admission rate to hospitals of patients with HIV/AIDS with numerous complications suggesting poor quality care at home (Mohammad \& Gikonyo, 2011). The re-admission rate for the other conditions is not known because the Health Management Information System (HMIS) does not capture data on re-admissions. The high re-admission rates of patients to hospitals may be due to many factors including the discharge health information given to them which could be insufficient to appropriately manage their self care at home. Little is known about research on assessing patients' informational needs in Malawi. Therefore, this study was conducted to assess patients'perceptions of the hospital discharge information at Chiradzulu District Hospital in Malawi.

\section{Methods}

\section{Design, setting and sampling}

This was a cross-sectional study that used qualitative research methods to gather data from participants. The study was conducted at Chiradzulu District Hospital which is a secondary referral hospital for 11 health facilities. The study participants included discharged adult patients who were admitted to the hospital due to medical and surgical conditions. The study also included 
patient guardians to discharged patients because they are the providers of patient care at home and qualified nurses and clinicians because they are the providers of information. Patients who were less than 18 years old and with conditions other than medical and surgical were excluded from the study. Guardians who were less than 18 years old were also excluded from the study. Written informed consent was granted from all participants included in the study. Through convenient sampling techniques, a total of 55 participants were recruited in this study (30 patients, 15 patient guardians and 10 health workers). The sample size of 30 patients and 15 patient guardians was determined based on a recommendation of twelve to more data sources when trying to achieve maximum variation (Kuzel, 2012). This study achieved maximum variation at the sample size of 30 patients and 15 patient guardians. A sample size of 10 health workers (5 nurses and 5 clinicians) was determined based on the rules of the thumb based on data collection method which recommend interviewing approximately five key informants to reach saturation (Patton, 2001). All these participants were involved in the in-depth interviews. The Malawi College of Medicine Research Committee granted ethical approval, while clearance was obtained from hospital authority.

\section{Data collection, processing and analysis}

Interviews were conducted at the hospital and patients were interviewed in the absence of their guardian. Interview guides were used during the in-depth individual interviews. All interviews were done in the local language (Chichewa) and were tape recorded. Each interview lasted approximately 20 minutes. The researcher reviewed the recorded data after the interviews were finished. All interviews were tape-recorded and verbatim responses to each question were translated into English and transcribed by the researcher and research assistants. Thematic analysis using QRS Nvivo 9 software was performed. An initial codebook was developed using a standard iterative process (McEllan et al., 2003). The codebook was revised as necessary during data analysis. Responses were summarized based on the set question and objectives.

Use of QRS Nvivo 9 software helped to establish an accurate and transparent audit trail and added rigor to the study.

\section{Findings}

\section{Demographic characteristics of the sample}

Out of 55 participants involved in the in-depth interviews, 30 were patients, 15 were patient guardians and 10 were health workers (nurses and clinicians). Fifteen out of 30 patients were men with mean age (years) 35.4 (SD 12.0). The highest level of education among the men was tertiary level although $7 \%(95 \% \mathrm{CI}=0.06,0.2)$ of these male participants did not attend 
formal education. The mean age (years) among females was 37 (SD 16.3). The highest level of education among female participants was secondary level while $27 \%(95 \% \mathrm{CI}=0.05,0.49)$ of the female participants did not attend formal education. All the 15 patient guardians were females with mean age (years) of 43.2 (SD 11.3). Their highest level of education was secondary education. Thirty percent $(95 \% \mathrm{CI}=0.07,0.53)$ of the patient guardians did not attend formal education. The length of hospital stay for patients was within the range of 5-30 days. Eighty percent $(95 \% \mathrm{CI}=0.66,0.94)$ of the patients had a history of previous admission to hospital. Of the 10 health workers, 5 were nurses and 5 were clinicians.

\section{Discharge information given to patients by nurses and clinicians}

Some patients and patient guardians explained that they were given information at discharge by nurses and clinicians. They were particularly given information on how to manage their care at home which included compliance to treatment, side effects of drugs, nutrition, follow up care and wound care. One patient guardian said this:

"A nurse has advised me to be giving medications to the patient at home every morning soon after the patient takes breakfast and that I should observe the patient taking the drugs. I also have been advised to encourage the patient to continue taking drugs even when the patient experiences some side effects as most of the side effects are not harmful and that they cease after some time. I also have been advised to be giving the patient nutritious foods at home", IDI_ G

When asked, nurses and clinicians explained that they try to provide patients and their guardians with some information on discharge from the hospital to enable them manage self-care at home appropriately.

Patients and patient guardians who were not given information at discharge expressed their discontentment since they wanted information about the conditions they were suffering from and how to manage their conditions at home. One patient said this:

"I am going home not knowing what I am suffering from because neither a nurse nor a clinician has told me about it. I am just thinking that maybe it is blood pressure because I have heart palpitations and I sweat excessively. I came to seek help from the hospital so they would have told me what is happening in my body”, IDI_FP

It was noted that nurses and clinicians fail to disseminate information to patients adequately mainly due to shortage of staff at the hospital. There 
were few nurses and clinicians taking care of large numbers of patients in the hospital wards where the study was conducted. One nurse said this:

"We are the two nurses taking care of all these patients. Some are sitting there on the chairs waiting for us to admit them, some are waiting to be discharged and others are very sick that they need total nursing care so how can we find time to give discharge information to each and every patient? ", IDI_N.

\section{Patients perceptions of discharge information \\ Patient perceptions of the relevance of discharge information}

Some patients and patient guardians indicated that the discharge information they were given was relevant. They pointed out that the information would assist them in performing self care at home. They particularly indicated that the information would assist them in managing side effects of drugs, meeting their nutritional requirements, preventing recurrences of their conditions, and managing their surgical incisions. Some indicated that they would strictly follow the instructions they were given by nurses and clinicians to recover quickly from the conditions they were suffering from. Some patients explained that the information brought them encouragement at the time they had worries over the conditions they were suffering from.

Those patients who indicated that the information they received was not relevant explained that the information was neither clear nor detailed as such they failed to understand it. One patient said this:

"The information that I am given on home care is not comprehensive. In fact, I failed to understand what the clinician wanted to communicate to me. The clinician said I should go home otherwise I would be wasting time to be in the hospital. I did not understand this because I came to the hospital to be assisted. Unless I understood what the clinician said, the information was not important", IDI_MP

\section{Patients perceptions of the adequacy of discharge information Patients informational needs}

It was clear from all the interviews with patients that they wanted information about their conditions and how to manage them at home. Some patients and patient guardians indicated that they were not satisfied with the information they received from the nurses and clinicians because it was not adequate. Some patients indicated that the information they received did not help them understand the treatment they were supposed to continue taking in their homes. Other patients desired information on the conditions they were suffering from. They wanted to be told their diagnoses and etiologies. Other patients desired to know the test results from the diagnostic tests that were 
carried out on them. Nurses and clinicians did not provide them with test results at the time they were leaving the hospital.

Some patients who were suffering from chronic medical conditions explained that health care workers (nurses and clinicians) indicated to them the role food would play in promoting their health. However, they did not explain into detail the foods that these patients required. The health care workers only explained that these patients required balanced diets. These patients explained that they wanted information on the examples of foods that would help promote their health as they did not know how to come up with a balanced diet. Also, other patients with chronic conditions (particularly those with HIV/AIDS) were discharged from the hospital while they were still very sick. These patients and their guardians complained that the clinicians did not explain to them why they were discharged in such conditions and also how to manage home care. They thought they were discharged so that they could die in their homes and not in the hospital.

Some patients who had undergone surgical procedures and guardians to these patients indicated that they needed information on a number of areas. These areas were wound dressing, time to resume normal activities, nutrition, pain management and signs of wound infection.

\section{Health care workers' perceptions of discharge information}

The providers of information, nurses and clinicians, indicated that they were aware that the information they were giving patients and patient guardians at discharge from hospital was not adequate when they were asked about their perceptions of this information. They explained that due to shortage of staff they could not find adequate time to provide the information to every patient and patient guardian as was expected but they were trying to provide adequate information to those patients who were suffering from chronic conditions like TB, HIV/AIDS and cancer. They indicated that they were aware that managing the condition at home could be difficult if the patients and patient guardians were not given adequate information on home care.

\section{Patients understanding of discharge information}

When asked about their understanding of the information they received from nurses and clinicians some patients and patient guardians reported that they understood the information and others did not understand the information. It was observed that several factors played a role in their understanding and not understanding of the information. 


\section{Factors that facilitate understanding of information}

Patients and patient guardians who understood the information outlined three factors that helped them to understand the information. These factors were clear explanation of information, use of understandable language by information providers (nurses and clinicians) and adequate time taken by the information providers to deliver the information.

\section{Factors that hinder patient understanding of information}

Patients and patient guardians who explained that they did not understand the information cited a number of factors that hindered their understanding of information. These factors were inadequate information by information providers (nurses and clinicians), lack of encouragement from health care workers to make them speak, fear of being shouted at by health care workers (because of the unfriendly attitude portrayed), fear of being seen as difficult and lack of privacy (as health care workers could discuss with patients sensitive information in the wards in the presence of other people). One patient said this:

"I failed to concentrate because the clinician was giving me sensitive information on the bedside where others were hearing. I was so uncomfortable because I did not want others to listen to the information. He would have found a private place where he would have given me the information", IDI_MP

\section{Patient perceptions of the method used in delivering the information}

All patients and patient guardians who received discharge information explained that it was given verbally. When asked about their views about the method of delivering information, the majority of patients and patient guardians said it was good in that each time they went to the hospital, nurses and clinicians used it so they were used to it.

Some patients and patient guardians indicated that although this was the method that was being used by nurses and clinicians to give information and that they were used to it, it had one major challenge. They explained that it was easy to forget particularly when one was given too much information. One patient said this:

"I have been given a lot of instructions by the nurse on the things I should be doing at home. Chances are high that I may forget some of the instructions. May be the nurse would have written down some of the instructions in my health passport booklet so I could be referring to them at home”, IDI_MP 


\section{Areas to be considered when providing discharge information}

When asked about the areas they thought nurses and clinicians should improve in order to provide information effectively, patients and patient guardians outlined a number of these areas. Also, health care workers outlined a number of areas they thought they needed to improve to provide information effectively.

\section{Ensuring patient understanding of information}

Some patients and patient guardians received too much information at discharge. There were chances that they could forget some of the information. These patients and patient guardians indicated that health care workers would have made sure that they could not forget any of the information because that would have a negative effect on home care. One patient said this:

"I think nurses and clinicians should be able to assess patients understanding of the information they receive. They should not assume that patients have understood and memorized the information. I have been given a lot of instructions on home care but the nurse did not find out if understood and remembered them all", IDI_MP

Nurses and clinicians verified that patients and guardians understanding of information was not being assessed by indicating that they rarely assess patients and patient guardians understanding of the information that has been given to them. They explained that some patients and their guardians, particularly those with chronic conditions, were being given a lot of information and assessing their understanding of the information would be of much help in that they could be reminded about the information they may have forgotten. As such patients and patient guardians would be able to utilize the information effectively in home care management.

All patients and patient guardians who received the information were not given chances by health care workers to ask questions on the information they were given. Some of these patients and patient guardians indicated that they had questions to ask on the information they received and that they would have asked questions if they were given chances. They also indicated that they would have asked about the information they wanted to know that was not explained by the health care workers if they were given chances to ask questions. There were a number of reasons that prevented patients and patient guardians to ask nurses and clinicians questions on the information that they received. These reasons were fear of being shouted at, not wanting to take much of the nurses and clinicians time as they had a lot of patients to assist and that nurses and clinicians were the ones who knew their conditions better. 
Some health care workers also pointed out that they do not give patients and their guardians' chances to ask questions on the information they receive. They indicated that this is one of the areas they needed to consider if they were to give information to their patients and their guardians' effectively.

Nurses and clinicians also did not summarize the information given to patients and their guardians. Those patients and patient guardians who received much information indicated that nurses and clinicians should consider summarizing the information to enable them remember some of the information that they may have forgotten.

\section{Patient involvement in discharge planning}

The study revealed that patients and their guardians were not involved in discharge planning. Patients and their guardians felt that it was necessary that they should have been involved in discharge planning as this would have provided nurses and clinicians with an opportunity to understand the information that they expected as they were going home to continue with self care.

Nurses and clinicians also indicated that there is need to involve patients particularly those with major conditions and their guardians in discharge planning. They explained that patients and patient guardians' worries or concerns could be resolved during discharge planning but due to pressure of work they fail to do so.

\section{Coordination among care providers}

Some patients reported lack of coordination amongst nurses and clinicians. They explained that these health care workers could sometimes not consult each other and they ended up giving conflicting information that made patients to become confused as they did not know which information was true. One patient said this:

"I am surprised that this clinician has discharged me because another clinician said that he would be coming to perform a procedure on my body in the afternoon. When I told him that his colleague said he would be coming to do a procedure he said that it was not necessary that the procedure should be done now. I do not know who to believe between the two. I would have loved if the clinician who has discharged me from hospital consulted his colleague so that they would agree on one thing. I am going home disappointed because of this", IDI_MP

Some health care workers also indicated that coordination is lacking amongst them, particularly between the nurses and clinicians. They explained 
that nurses and clinicians are supposed to be working together during ward rounds but this rarely happens. They pointed out that in such situations nobody takes the responsibility of providing discharge information to the patients and their guardians.

\section{Update of knowledge}

Some health care workers pointed out that they sometimes fail to provide adequate information to some patients and their guardians because they have limited knowledge on the conditions the patients are suffering from. They reported lack of reading culture and lack of good books in the institution's library as some of the factors leading to failure to update their knowledge on some of the conditions. One nurse said this:

"Sometimes we fail to give adequate information to patients and their guardians because we have little knowledge of the conditions they are suffering from. I for one, become so uncomfortable to provide education to a diabetic patient because I have little knowledge of this condition", IDI_N

\section{Use of Information Education and Communication (IEC) materials}

Some health care workers explained that the majority of patients and patient guardians had difficulties in understanding the information. One of the reasons for this was because they have not gone far with their education. They (health care workers) pointed out that information could be delivered effectively to these patients if health care workers are using the available IEC materials to explain their conditions and how they can manage their care at home.

\section{Discussion of findings}

The results of this study validate the importance of discharge teaching in preparing patients and their guardians to appropriately manage homecare. The findings show that discharge teaching places the patients and their guardians in a state of readiness that sets the stage for successfully managing care and continuing recovery at home.

The results of this study made it clear that discharged patients' and their guardians' have unmet information needs. Patients and their guardians desire information on diet, wound care, signs and symptoms to watch for on the wound, time to resume normal activities following surgery, pain management and the diseases they are suffering from and their treatment. A number of studies are consistent with the findings of this study that patients received inadequate information on diet (Jacobs, 2008; Henderson \& Zernike, 2001), wound care (Forster et al., 2003, Henderson \& Zernike, 2001), time to resume normal activities after surgery (Forster et al., 2003; LeClerc et al., 
2002; Sheikh et al., 1993), pain management (Boyle et al., 1992) and diseases that patients' were suffering from and their treatment (Selman, 2011). Adequate information should be given to patients and their guardians because information appears to have empowering effect, enabling patients and their guardians to take control over their health and comply with treatment (Cortis \& Lacey, 1996).

Availability of information providers (nurses and clinicians) is one of the critical issues in this study. Shortage of nurses and clinicians has a negative impact on discharge teaching. The few nurses and clinicians available in the hospital do not have enough time to provide information to patients and their guardians. This is consistent with an observation that was made by one of the studies that due to shortage of health care workers in hospitals there is less time available to educate patients about post-hospital care (McMurray et al., 2007).

Overloading patients and their guardians with information is another critical area that is arising in this study. This interferes with retention of information. Anxiety, fatigue and feelings of going home can all potentially impact attention and retention of content presented in discharge teaching. Identification of information to meet individualized needs may reduce the amount of content given to patients and their guardians.

Another important area in this study relates to the assessment of patients and guardians understanding of discharge information given to them by nurses and clinicians. Assessing patients and guardians understanding of information provides an opportunity for reminding them the information they may have forgotten that would assist them in managing homecare appropriately. There are several ways nurses and clinicians can use to assess patients and guardians understanding of information. These methods include asking questions or asking them to explain the information they have been given. In addition to assessing patients and guardians understanding of information, they should be encouraged to speak because this may motivate them to ask about the information they perceive to be important from nurses and clinicians. In this study, patients'and guardians' failure to ask nurses and clinicians to give them the information they wanted was another factor that had a negative effect on discharge information. Nurses and clinicians gave patients' and guardians' the information that they themselves perceived as important. One explanation for patients and guardians failure to ask nurses and clinicians the information they wanted would be that the majority of the patients and guardians have grown up in a culture where it is considered impolite to ask questions from someone who is considered senior. Nurses and clinicians should therefore, not expect that patients will assume the responsibility for their health by asking questions to solicit the information they need. 
The other important finding in this study relates to the importance of involving patients and their guardians in discharge planning. Patients and their guardians should not be passive recipients of information. They should be central within the discharge planning process because this has such beneficial effects as increased patient satisfaction with care and communication, increased certainty about making the best decisions, reduced anxiety levels and greater adherence to chosen management plans (Holland et al., 2003). Excluding patients and guardians from discharge planning results in failure to ascertain and address patients and guardians informational needs. Patients who are discharged with unmet informational needs are at risk for posthospitalization complications and readmission. The finding in this study on lack of involvement of patients and their guardians in the discharge planning process is consistent with the findings of a study in which patients and their caregivers often felt excluded from discharge planning as such they were not well prepared for what life would be like after discharge and their first days at home were characterized by uncertainty, anxiety and dejection (Tierney et al. 1993).

\section{Strengths and limitations of the study}

Much as the views of the health care workers (nurses and clinicians) were sought which strengthened the findings of the study, this study had some limitations. Firstly, data for this study were collected in a single hospital and may not reflect the experiences of patients in other hospitals. Secondly, the data on which study findings were based centered on patients' and guardians' perceptions which reflected their reality but may not have accurately represented the actual teaching that was provided. Despite these limitations, the study provides interesting preliminary findings that deserve further exploration/investigation.

\section{Conclusion}

It has been noted from this study that patients and their guardians have a variety of information needs at discharge from hospital. The findings of this study have revealed that most patients and their guardians perceive the discharge information provided by nurses and clinicians as inadequate. They explained that they needed additional discharge information to that which they were given to appropriately manage self-care at home. The study has also revealed that patients and their guardians are not involved in discharge planning. The main reason to these problems has been found to be lack of time that nurses and clinicians experience because they are in short supply. We therefore, recommend that nurses and clinicians should be able to determine patients and patient guardians understanding of discharge information. Nurses 
and clinicians should also involve patients and their guardians during discharge planning to identify the information that they need.

\section{References:}

1. Boyle, K., Nance, J., \& Passau-Buck, S. (1992). Post-hospitalization concerns of medical-surgical patients. Applied Nursing Research, 5, 122-126.

2. Cortis, J.D., \& Lacey, A.E. (1996). Measuring the quality and quantity of

3. information-giving to in-patients. Journal of Advanced Nursing, 24, 674-681.

4. Forster, H.J., Murff, J.F., Peterson, T.K., Gandhi, G. \& Bates, D.W. (2003).

5. The incidence and severity of adverse events affecting patients after discharge from the hospital. Annals of Internal Medicine, 13, 161-167.

6. Henderson, A., \& Zernike, W. (2001). A study of impact of discharge

7. information for surgical patients. Journal of Advanced Nursing, 35(3), 435-441.

8. Holland, D., Harris, M., Pankratz, S., Closson, D., Matt-Hensrud, N., \&

9. Severson, M. (2003). Prospective evaluation of a screen for complex discharge planning in hospitalized adults. Journal of American Geriatric Society, 51, 678-682.

10. Jacobs, V. (2000). Informational needs of surgical patients followingdischarge. Applied Nursing Research, 13(1), 12-18.

11. Kuzel, A (2012, January, 8). Sampling in qualitative inquiry, in doing

12. qualitative research. Retrieved 12 February, 2012 from: http://www.springer.com/chapter/10.700\%2F978-3-642.

13. LeClerc, C.M., Wells, D., Graig, D., \& Wilson, J.L. (2002). Falling short of

14. the mark: tales of life after hospital discharge. Clinical Nursing Research, 11(3), 243-263.

15. McEllan, E., MacQueen, K.M., \& Niedig, J. (2003). Beyond the qualitative

16. interview: data preparation and transcription. Field Methods, 15(1), 63-84.

17. McMurray, A., Johnson, P., Wallis, M., Patterson, E., \& Griffiths, S. (2007).

18. General surgical patients' perspectives of the adequacy and appropriateness of discharge planning to facilitate health-decision making at home. Journal of Clinical Nursing, 16, 1602-1609. 
19. Meleis, A.L., Sawyer, L.M., ImE, O., Messias, D.K.H., \& Schumacher, K.

20. (2000). Experiencing transitions: an emerging middle-range theory. Advances in Nursing Science, 23(1), 12-28.

21. Meleis, A.L., \& Trangestein, P.A. (1994). Facilitating transitions: redefinition of the nursing mission. Nursing Outlook, 42(6), 255-259.

22. Mohammad, N., \& Gikonyo, J. (2011, June 22). Operational challenges:

23. community home-based care for PLWHA in multi-country HIV/AIDS Programmes (MAP) for sub-Saharan Africa. Retrieved 15 March, 2012 from http://www.wds.worldbank.org/servelt/WDSContentServer/WDSP/I B/2005/11/17/000090341_20051117135435/Rendered/INDEX/3424 90PAPEROOP1I0.

24. Patton, M. Q. (2001). Qualitative evaluation and research methods $\left(3^{\text {rd }}\right.$ ed.). Newbury Park: Sage Publications.

25. Selman, L. (2011, April 14). Palliative care skills training needed for health

26. care staff in sub-Saharan Africa. Retreaved March 15, 2012 from http://www.sciencedaily.com/releases/2009/04/09042213737.htm

27. Sheikh, L., O’Brien, M., \& McCluskey-Fawcett, K. (1993). Parent

28. preparation for the NICU-to-home transition: Staff and patient perceptions. Children's Health Care, 22, 227-239.

29. Smith, J., \& Liles, C. (2007). Information needs before discharge of

30. myocardial infarction patients: a comparative, descriptive study. Journal of Clinical Nursing, 16, 662-667.

31. Tierney, A.J., Closs, S.J., Hunter, H.C., \& MacMillan, M.S. (1993).

32. Experiences of elderly patients concerning discharge from hospital. Journal of Clinical Nursing, 2, 179-185. 